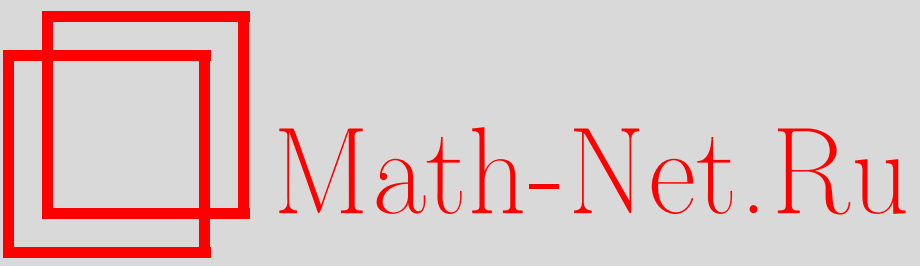

М. И. Купцов, В. А. Минаев, М. С. Маскина, Метод функций Ляпунова в задаче устойчивости интегрального многообразия системы обыкновенных дифференциальных уравнений, Итоги науки и техн. Сер. Соврем. мат. и ее прил. Темат. обз., 2020, том 186, 74-82

DOI: https://doi.org/10.36535/0233-6723-2020-186-74-82

Использование Общероссийского математического портала Math-Net.Ru подразумевает, что вы прочитали и согласны с пользовательским соглашением

http://www.mathnet.ru/rus/agreement

Параметры загрузки:

IP: 54.80 .73 .141

26 апреля 2023 г., 11:59:02 


\title{
МЕТОД ФУНКЦИЙ ЛЯПУНОВА В ЗАДАЧЕ УСТОЙЧИВОСТИ ИНТЕГРАЛЬНОГО МНОГООБРАЗИЯ СИСТЕМЫ ОБЫКНОВЕННЫХ ДИФФЕРЕНЦИАЛЬНЫХ УРАВНЕНИЙ
}

\author{
(c) 2020 г. М. И. КУПЦОВ, В. А. МИНАЕВ, М. С. МАСКИНА
}

\begin{abstract}
АннотАция. Рассматривается задача устойчивости ненулевых интегральных многообразий нелинейной конечномерной системы обыкновенных дифференциальных уравнений, правая часть которой является периодической вектор-функцией по независимой переменной и содержит параметр. Предполагается, что у изучаемой системы имеется тривиальное интегральное многообразие при всех значениях параметра, а соответствующая линейная подсистема не обладает свойством экспоненциальной дихотомии. Целью работы является нахождение достаточных условий устойчивости, неустойчивости и асимптотической устойчивости локального ненулевого интегрального многообразия. Для этой цели применяется метод функций Ляпунова, модифицированный к рассматриваемой задаче и особенностям правых частей изучаемой системы дифференциальных уравнений.
\end{abstract}

Ключевъе слова: метод функций Ляпунова, устойчивость, асимптотическая устойчивость, неустойчивость, интегральное многообразие, система обыкновенных дифференциальных уравнений.

\section{METHOD OF LYAPUNOV FUNCTIONS IN THE PROBLEM OF STABILITY OF INTEGRAL MANIFOLDS OF A SYSTEM OF ORDINARY DIFFERENTIAL EQUATIONS}

\author{
(c) 2020 M. I. KUPTSOV, V. A. MINAEV, M. S. MASKINA
}

\begin{abstract}
We consider the problem of stability of nonzero integral manifolds of a nonlinear finitedimensional system of ordinary differential equations whose right-hand side is a periodic vector-valued function of the independent variable containing a parameter. We assume that the system has a trivial integral manifold for all values of the parameter and the corresponding linear subsystem does not possess the property of exponential dichotomy. The aim of this work is to find sufficient conditions for stability, instability, and asymptotic stability of a local nonzero integral manifold. For this purpose, we use the method of Lyapunov functions modified to the problem considered and singularities of the right-hand sides of the system.
\end{abstract}

Keywords and phrases: method of Lyapunov functions, stability, asymptotic stability, instability, integral manifold, system of ordinary differential equations.

AMS Subject Classification: 34A34, 34C25, 34C45, 34D35

1. Введение. Пусть система обыкновенных дифференциальных уравнений

$$
f(\nu, y, \dot{y}, t)=0
$$

для любых $\nu, t$ имеет состояние равновесия $y=0$ и в области $\Lambda$ ее решения существуют и единственны. Здесь и далее $f, y, \nu-(n+m)$-векторы, $\dot{y}=\frac{d y}{d t}, f(\nu, y, \dot{y}, t+T) \equiv f(\nu, y, \dot{y}, t), \Lambda=\Lambda_{1} \times \Lambda_{2}$, 
$\Lambda_{1}=\left\{y:\|y\| \leqslant \Delta_{1}\right\} \subset \mathbb{R}^{n+m}, \Lambda_{2}=\left\{\nu:\|\nu\| \leqslant \Delta_{2}\right\} \subset \mathbb{R}^{n+m}, \Delta_{1}, \Delta_{2}$ - константы, $\mathbb{R}^{p}$ - стандартное евклидово пространство размерности $p$ и $\|\cdot\|$ - евклидова норма в $\mathbb{R}^{p}$.

Предположим, что замена переменных

$$
y=\Gamma(\varepsilon, x, \phi, t), \quad \nu=\xi(\varepsilon)
$$

систему (1) приводит к виду

$$
\left\{\begin{array}{l}
\dot{x}=X(\varepsilon, x, \phi, t) \cdot x, \\
\dot{\phi}=\Phi(\varepsilon, x, \phi, t),
\end{array}\right.
$$

где $\Gamma, \xi, \varepsilon-(n+m)$-векторы, $X-(n \times n)$-матрица, $x-n$-вектор, $\phi, \Phi-m$-векторы. Кроме того, будем полагать, что система

$$
\left\{\begin{array}{l}
\dot{x}=X(0,0, \phi, t) \cdot x, \\
\dot{\phi}=\Phi(0,0, \phi, t),
\end{array}\right.
$$

имеет $m$-параметрическое семейство ненулевых $k T$-периодических решений $x=\bar{x}\left(\phi_{0}, t\right), \phi=$ $\bar{\phi}\left(\phi_{0}, t\right)$.

Определение 1. Будем говорить, что у системы (1) существует $n$-мерное нетривиальное периодическое интегральное многообразие $\Psi\left(\phi_{0}, t\right)$, если для всех $\phi_{0}$ найдется такое значение $\nu_{0}=\xi\left(\varepsilon_{0}\right)$ параметра $\nu$, при котором

$$
f\left(\xi\left(\varepsilon_{0}\right), \Gamma\left(\varepsilon_{0}, \Psi\left(\phi_{0}, t\right), \phi^{\Psi}\left(\phi_{0}, t\right), t\right), \dot{\Gamma}\left(\varepsilon_{0}, \Psi\left(\phi_{0}, t\right), \phi^{\Psi}\left(\phi_{0}, t\right), t\right), t\right) \equiv 0,
$$

причем $\Psi\left(\phi_{0}, t\right)$ не обращается в ноль ни при каких значениях $\phi_{0}$ и $t$, является $\omega$-периодическим по компонентам $m$-вектора $\phi_{0}, k T$-периодическим по $t$, где $k$-натуральное число, $\omega=\operatorname{colon}\left(\omega_{1}, \omega_{2}, \ldots, \omega_{m}\right), \phi=\phi^{\Psi}\left(\phi_{0}, t\right)$ определяет интегральную кривую на многообразии.

Ставятся задача получения достаточных условий устойчивости, неустойчивости и асимптотической устойчивости нетривиального периодического $n$-мерного интегрального многообразия системы (1) вблизи нулевого многообразия $x=0$.

Современный этап развития информационных технологий тесно связан с увеличением применения элементов искусственного интеллекта, возникновением и совершенствованием различных направлений анализа больших данных и других систем, в основе которых лежат математические алгоритмы и модели. Поэтому математические модели и алгоритмы становятся сложнее, описывая все более широкий класс прикладных задач в различных областях: от технических и экономических до лингвистических и поведенческих. Важную роль в построении моделей всевозможных природных, социальных и других процессов и явлений играют дифференциальные уравнения. Изучение указанных явлений предполагает и исследование инвариантных множеств у описывающих их систем дифференциальных уравнений. Потребность в таких исследованиях обусловлена как широтой и значимостью практических приложений инвариантных множеств, так и использованием их для решения целого ряда теоретических проблем самой математики.

Появление методов изучения инвариантных множеств нелинейных систем дифференциальных уравнений связано прежде всего с трудами А. Пуанкаре и А. М. Ляпунова. Развитие электрои радиотехники привело к формированию теории нелинейных колебаний, а ее постепенное усложнение - к теории многочастотных колебаний. В основе указанных теорий лежат исследования различных инвариантных множеств систем дифференциальных уравнений: от особых точек до странных аттракторов и интегральных многообразий достаточно общей природы. На настоящий момент получены обширные результаты как для обыкновенных дифференциальных уравнений, так и для дифференциальных уравнений в частных производных (см., например, $[5,11,20,21]$ ), достаточно подробный обзор литературы по которым можно найти в работах $[8,16,17]$.

$\mathrm{K}$ наиболее продуктивным методам изучения интегральных многообразий следует отнести метод малого параметра [4], точечных отображений [14], усреднения [12] и метод интегральных многообразий. Метод интегральных многообразий, разработанный Н. Н. Боголюбовым, Ю. А. Митропольским и А. М. Самойленко $[2,13,16]$, состоит в построении функции Грина и успешно применяется для многих систем вида (3)(см., например, $[11,20])$. В большей части работ, в которых используется данный метод, заменой (2)(см., например, [22]) уравнение (1) сводится к системе (3) 
так, чтобы правая часть первых $n$ уравнений не обращалась тождественно в ноль при $x=0$. Затем на основе функции Грина уравнения $\dot{x}=X(0,0, \phi, t) \cdot x$ строится нелинейный оператор, неподвижные точки которого и являются интегральным многообразием системы (1). Однако в данном случае этот подход реализовать не удается, поскольку система (3) при всех значениях параметра имеет нулевое интегральное многообразие $x=0$, а система (4) - параметрическое семейство периодических решений. Указанные условия удается обойти лишь с помощью нахождения решения вспомогательного векторного (бифуркационного) уравнения и перехода в его окрестность $[1,3]$. При этом проблема устойчивости полученного многообразия решается за счет ограниченности функции Грина, т. е. фактически предполагается, что матрица $X(0,0, \phi, t)$ такова, что допускает необходимую оценку.

Изложенные в данной статье результаты получены на основе модификации метода функций Ляпунова, с помощью которого получены новые достаточные условия существования устойчивого, неустойчивого и асимптотически устойчивого локального интегрального многообразия системы (1). Полученные результаты развивают и обобщают работы [6-10, 18, 19, 22].

2. Существование интегрального многообразия. Пусть $F(\phi, t) \in \Omega_{1}, \varepsilon(\phi) \in \Omega_{2}-\omega$-периодические по компонентам вектора $\phi$ ограниченные соответственно числами $\delta_{10}$ и $\delta_{20}$ векторфункции, удовлетворяющие условию Липшица:

$$
\begin{gathered}
\left\|F\left(\phi_{1}, t_{1}\right)-F\left(\phi_{2}, t_{2}\right)\right\| \leqslant \delta_{11}\left\|\phi_{1}-\phi_{2}\right\|+\delta_{12}\left|t_{1}-t_{2}\right|, \\
\left\|\varepsilon\left(\phi_{1}\right)-\varepsilon\left(\phi_{2}\right)\right\| \leqslant \delta_{21}\left\|\phi_{1}-\phi_{2}\right\|,
\end{gathered}
$$

имеющие соответственно размерность $n$ и $l, 0<l \leqslant n+m, F(\phi, t)-k T$-периодическая по $t$,

$$
\|F(\phi, t)\|=\left[\sum_{i=1}^{n} \sup _{\substack{\phi_{j} \in\left[0, \omega_{j}\right] \\ t \in[0, k T]}}\left|F_{i}(\phi, t)\right|\right]^{1 / 2}, \quad\|\varepsilon(\phi)\|=\left[\sum_{i=1}^{l} \sup _{\phi_{j} \in\left[0, \omega_{j}\right]}\left|\varepsilon_{i}(\phi)\right|\right]^{1 / 2} .
$$

Отметим, что если для множеств $\Omega_{i}$ ввести указанную норму, то они становятся выпуклыми компактами [8, с. 15].

Для решения дифференциального уравнения

$$
\dot{\phi}=\Phi\left(\varepsilon\left(\phi_{0}\right), F\left(\phi_{0}, t\right), \phi, t\right),
$$

удовлетворяющего начальным данным $\phi(0)=\phi_{0}$, примем обозначение $\phi_{t}^{F}$. Пусть, кроме того, $Y_{\varepsilon}^{F}\left(\phi_{0}, t\right)$ - матрицант уравнения

$$
\dot{x}=X\left(\varepsilon\left(\phi_{0}\right), F\left(\phi_{0}, t\right), \phi_{t}^{F}, t\right) \cdot x .
$$

Здесь и далее $F\left(\phi_{0}, t\right) \in \Omega_{1}, n+m-l$ значений компонентов вектора $\varepsilon$ приняты равными 0 , а вместо оставшихся $l$ значений в уравнения системы (3) подставлены элементы функции $\varepsilon\left(\phi_{0}\right) \in \Omega_{2}$.

Определение 2 . Неособенную функциональную $n \times n$-матрицу $Q_{\varepsilon}^{F}\left(\phi_{0}\right)$ с постоянным определителем, непрерывную по всем своим переменным и $\omega$-периодическую по компонентам вектора $\phi_{0}$, будем называть преобразующей матрицей системы (3), если у матрицы

$$
\left(Y_{\varepsilon}^{F}\left(\phi_{0}, k T\right)-I_{n}\right) \cdot Q_{\varepsilon}^{F}\left(\phi_{0}\right)
$$

существует столбец $q_{\varepsilon}^{F}\left(\phi_{0}\right)$, тождественно в ноль не обращающийся. Здесь $I_{n}-$ единичная $n \times n$ матрица.

Обозначим $\mathrm{X}_{n}=\left\{x:\|x\| \leqslant \delta_{1}\right\} \subset \mathbb{R}^{n}, \mathrm{E}=\left\{\varepsilon:\|\varepsilon\| \leqslant \delta_{2}\right\} \subset \mathbb{R}^{n+m}, \Theta=\left\{\phi: \phi_{j} \in\left[0, \omega_{j}\right]\right\}$ и пусть $\Gamma(\varepsilon, x, \phi, t) \neq 0$ при $x \neq 0$,

$$
\begin{aligned}
& \Gamma(\varepsilon, 0, \phi, t) \equiv 0, \\
& \Gamma(\varepsilon, x, \phi, t) \rightarrow 0,
\end{aligned}
$$

при $\|x\| \rightarrow 0$ равномерно относительно совокупности переменных $\varepsilon, \phi, t$ в области $\mathbb{R}^{m+1} \times \mathrm{X}_{n} \times \mathrm{E}$. 
Здесь и далее мы предполагаем, что правые части системы (3) являются $\omega$-периодическими по компонентам вектора $\phi$ и $T$-периодическими по независимой переменной $t \in \mathbb{R}$, непрерывны и обеспечивают существование и единственность решений системы (3) в области $\mathbb{R}^{m+1} \times \mathrm{X}_{n} \times$ Е при достаточно малых $\delta_{1}$ и $\delta_{2}$. Это, в частности, означает, что замена переменных (2) не только сохраняет свойства существования и единственности решений системы (1), но и является $\omega$-периодической по $\phi$ и $T$-периодической по $t$.

Введем в рассмотрение систему уравнений

$$
\left\{\begin{array}{l}
q_{\varepsilon}^{F}\left(\phi_{0}\right)=0, \\
\int_{0}^{k T} \Phi\left(\varepsilon\left(\phi_{0}\right), F\left(\phi_{0}, t\right), \phi_{t}^{F}, t\right) d t=0 .
\end{array}\right.
$$

Теорема 1. Пусть преобразующую матричу системь (3) удается построить так, что существуют такое число $l(0<l \leqslant n+m)$ и такой столбеи, $q_{\varepsilon}^{F}\left(\phi_{0}\right)$, при которых для нахождения решения системы (12) достаточно найти решение некоторой, вообще говоря, отличной от (12) системы l уравнений

$$
S_{\varepsilon}^{F}\left(\phi_{0}\right)=0
$$

имеющей для каждой функиии $F\left(\phi_{0}, t\right) \in \Omega_{1}$ единственное решение $\varepsilon^{F}\left(\phi_{0}\right)$ из множества $\Omega_{2}$. Кроме того, пусть при $t \in[0 ; k T]$ выполнено:

$$
\begin{gathered}
\left\|Y_{\varepsilon}^{F}\left(\phi_{0}, t\right) \cdot Q_{\varepsilon}^{F}\left(\phi_{0}\right)\right\| \leqslant r_{0}, \\
\left\|Y_{\varepsilon}^{F}\left(\phi_{0}^{*}, t^{*}\right) \cdot Q_{\varepsilon}^{F}\left(\phi_{0}^{*}\right)-Y_{\varepsilon}^{F}\left(\phi_{0}, t\right) \cdot Q_{\varepsilon}^{F}\left(\phi_{0}\right)\right\| \leqslant r_{1}\left\|\phi_{0}^{*}-\phi_{0}\right\|+r_{2}\left|t^{*}-t\right| .
\end{gathered}
$$

Тогда для любого вектора $\phi_{0} \in \mathbb{R}^{m}$ можно указать такое значение параметра $\nu$, что система (1) будет иметь ненулевое интегральное многообразие $\Psi\left(\phi_{0}, t\right) \in \Omega_{1}$ в окрестности состояния равновесия $y=0$.

Доказательство теоремы 1 опубликовано в $[10,19]$ и поэтому здесь мы его не приводим. Отметим лишь, что для интегрального многообразия $\Psi\left(\phi_{0}, t\right)$, существование которого и доказывается в теореме 1 , справедливо равенство

$$
\Psi\left(\phi_{0}, t\right)=Y_{\varepsilon}^{\Psi}\left(\phi_{0}, t\right) \cdot Q_{\varepsilon}^{\Psi}\left(\phi_{0}\right) \cdot C,
$$

где все элементы постоянного $n$-вектора $C$ равны нулю, кроме элемента, соответствующего номеру столбца $q_{\varepsilon}^{\Psi}\left(\phi_{0}\right)$, который равен $c$ - произвольной константе. При этом значения параметра $\varepsilon$ удовлетворяют уравнению (13), а, следовательно, и $(12)$, в которых $F\left(\phi_{0}, t\right) \equiv \Psi\left(\phi_{0}, t\right)$. Тогда выполнено $\lim _{c \rightarrow 0}\left(\left\|\Psi\left(\phi_{0}, t\right)\right\| / c\right) \neq \infty$ и $\lim _{c \rightarrow 0}\left\|\varepsilon^{\Psi}\left(\phi_{0}\right)\right\|=0$.

Техника построения преобразующей матрицы подробно рассмотрена в $[8,19]$.

3. Устойчивость и неустойчивость интегрального многообразия. Пусть теперь и далее выполнены условия теоремы 1 и, значит, у системы (1) при некоторых значениях параметра $\nu_{0}=\xi\left(\varepsilon_{0}\right)$ имеется интегральное многообразие $\Psi\left(\phi_{0}, t\right) \in \Omega_{1}, \Psi\left(\phi_{0}, 0\right)=\Psi_{0}$. Обозначим $x\left(x_{0}, \phi_{0}, t\right), \phi^{x}\left(x_{0}, \phi_{0}, t\right)$ - решение уравнения $(3)$, удовлетворяющее начальным данным $x\left(x_{0}, \phi_{0}, 0\right)=x_{0}, \phi^{x}\left(x_{0}, \phi_{0}, 0\right)=\phi_{0}$.

Пусть функция $V(x, \phi, t)$ имеет непрерывные частные производные по всем своим переменным в области $\mathbb{R}^{m+1} \times \mathrm{X}_{n}$ и $V(0, \phi, t) \equiv 0$ при $\phi \in \mathbb{R}^{m}, t \geqslant 0$.

Определение 3 (см. [15]). Функцию $V(x, \phi, t)$ будем называть $x$-определенно-положительной ( $x$-определенно-отрицательной), если существует определенно-положительная функция $W(x)$, для которой в области $\mathbb{R}^{m+1} \times \mathrm{X}_{n}$ выполняется неравенство

$$
V(x, \phi, t) \geqslant W(x)
$$

(соответственно $-V(x, \phi, t) \geqslant W(x))$.

Функцию $V(x, \phi, t)$ будем называть $x$-знакоопределенной, если она является $x$-определенноположительной или $x$-определенно-отрицательной.

Заметим, что в [15] полагается $V(0,0, t) \equiv 0$. 
Теорема 2. Если при $\varepsilon=\varepsilon_{0}$ существует $x$-знакоопределенная функиия $V(x, \phi, t)$, для которой функиия

$$
\begin{gathered}
\frac{d V}{d t}=\frac{\partial V\left(x\left(x_{0}, \phi_{0}, t\right)-\Psi\left(\phi_{0}, t\right), \phi^{x}\left(x_{0}, \phi_{0}, t\right)-\phi^{\Psi}\left(\Psi_{0}, \phi_{0}, t\right), t\right)}{\partial t}+ \\
+\sum_{i=1}^{n} \frac{\partial V\left(x\left(x_{0}, \phi_{0}, t\right)-\Psi\left(\phi_{0}, t\right), \phi^{x}\left(x_{0}, \phi_{0}, t\right)-\phi^{\Psi}\left(\Psi_{0}, \phi_{0}, t\right), t\right)}{\partial x_{i}}+ \\
+\sum_{j=1}^{m} \frac{\partial V\left(x\left(x_{0}, \phi_{0}, t\right)-\Psi\left(\phi_{0}, t\right), \phi^{x}\left(x_{0}, \phi_{0}, t\right)-\phi^{\Psi}\left(\Psi_{0}, \phi_{0}, t\right), t\right)}{\partial \phi_{j}}
\end{gathered}
$$

знакопостояннал, знака, противоположного $\kappa$ знаку $V(x, \phi, t)$, то при $\nu_{0}=\xi\left(\varepsilon_{0}\right)$ интегральное многообразие $\Psi\left(\phi_{0}, t\right)$ системы (1) устойчиво.

Доказательство. Пусть $\varepsilon=\varepsilon_{0}$ и $V(x, t)-x$-определенно-положительная и пусть $\delta>0$ - произвольное фиксированное число. Обозначим $\bar{l}=\inf _{\|x\|=\Delta} W(x)$, где $0<\Delta<\delta, \Delta<\delta_{1}$. Выберем теперь $x_{0}$ так, что

$$
\left\|x_{0}-\Psi_{0}\right\| \leqslant \bar{\Delta}<\Delta
$$

и

$$
V\left(x_{0}-\Psi_{0}, \phi_{1}-\phi_{2}, 0\right)<\bar{l}
$$

для любых $\phi_{1} \in \Theta, \phi_{2} \in \Theta$. В силу того, что

$$
\frac{d V}{d t} \leqslant 0
$$

неравенство

$$
\| x\left(x_{0}, \phi_{0}, t\right)-\Psi\left(\phi_{0}, t \|<\Delta\right.
$$

справедливо для всех $\phi_{0} \in \Theta$ при $t>0$. Действительно, если бы это было не так, то существовали бы такие постоянные $\phi_{0}^{*} \in \Theta$ и $t^{*}>0$, для которых выполнялось $\left\|x\left(x_{0}, \phi_{0}^{*}, t^{*}\right)-\Psi\left(\phi_{0}^{*}, t^{*}\right)\right\|=\Delta$ и, значит,

$$
V\left(x\left(x_{0}, \phi_{0}^{*}, t^{*}\right)-\Psi\left(\phi_{0}^{*}, t^{*}\right), \phi^{x}\left(x_{0}, \phi_{0}^{*}, t^{*}\right)-\phi^{\Psi}\left(\Psi_{0}, \phi_{0}^{*}, t^{*}\right), t^{*}\right) \geqslant W\left(x\left(x_{0}, \phi_{0}^{*}, t^{*}\right)-\Psi\left(\phi_{0}^{*}, t^{*}\right)\right) \geqslant \bar{l},
$$

что противоречит одновременному выполнению соотношений (20) и (21).

Таким образом, для любого числа $\delta>0$ существует такая постоянная $\Delta>0$, что выполнение неравенства (19) влечет выполнение неравенства (22). Тогда из соотношений (10) и (11) следует устойчивость интегрального многообразия $\Psi\left(\phi_{0}, t\right)$ системы $(1)$ при $\nu_{0}=\xi\left(\varepsilon_{0}\right)$.

Заметим, что в отличие от [15] устойчивость здесь подразумевается относительно всех переменных $(y)$, причем задача устойчивости полностью решается по свойствам лишь части переменных $(x)$.

Справедливы и общие теоремы об асимптотической устойчивости и неустойчивости интегрального многообразия. Схемы доказательства этих фактов в целом повторяют классические рассуждения (см., например, [15]) с некоторыми модификациями, обусловленными спецификой рассматриваемой системы (1).

Отметим, что если $V(x, \phi, t)$ допускает бесконечно малый высший предел по переменной $x$ (в смысле [15]), то $V(x, \phi, t) \rightarrow 0$ при $\|x\| \rightarrow 0$ равномерно по $t \geqslant 0$ и $\phi \in \mathbb{R}^{m}$.

Теорема 3. Если при $\varepsilon=\varepsilon_{0}$ существует $x$-знакоопределенная функиия $V(x, \phi, t)$, для которой функиия (18) $x$-знакоопределеннал, знака, противоположного к знаку $V(x, \phi, t)$, а сама функиия $V(x, \phi, t)$ допускает бесконечно малый высший предел по переменной $x$, то при $\nu_{0}=\xi\left(\varepsilon_{0}\right)$ интегральное многообразие $\Psi\left(\phi_{0}, t\right)$ системъ (1) устойчиво асимптотически.

Доказательство. Пусть $\varepsilon=\varepsilon_{0}$ и $V(x, \phi, t)$ - положительная. Тогда из $x$-определенно-отрицательности производной (18) следует справедливость неравенства (21), а, значит, выполнены условия 
теоремы 2 , и многообразие $\Psi\left(\phi_{0}, t\right)$ устойчиво. Значит, начальные данные $x_{0}$ можно выбрать настолько близкими к $\Psi_{0}$, что при $t \in[0 ;+\infty)$ выполнено $d V / d t<0$ (см. (18)) и

$$
V\left(x\left(x_{0}, \phi_{0}, t\right)-\Psi\left(\phi_{0}, t\right), \phi^{x}\left(x_{0}, \phi_{0}, t\right)-\phi^{\Psi}\left(\Psi_{0}, \phi_{0}, t\right), t\right)
$$

убывает.

Если предположить, что (23) ограничено снизу, то тогда

$$
\left\|x\left(x_{0}, \phi_{0}, t\right)-\Psi\left(\phi_{0}, t\right)\right\| \geqslant l_{1}>0,
$$

и, следовательно, $d V / d t \leqslant-l_{2},\left(l_{2}>0\right)$. Значит,

$$
\begin{aligned}
V\left(x\left(x_{0}, \phi_{0}, t\right)-\Psi\left(\phi_{0}, t\right), \phi^{x}\left(x_{0}, \phi_{0}, t\right)-\phi^{\Psi}\right. & \left.\left(\Psi_{0}, \phi_{0}, t\right), t\right)= \\
& =V\left(x_{0}-\Psi_{0}, 0,0\right)+\int_{0}^{t} \frac{d V}{d t} d t \leqslant V\left(x_{0}-\Psi_{0}, 0,0\right)-l_{2} t,
\end{aligned}
$$

что противоречит (24). Поэтому предположение об ограниченности снизу функции (23)неверно и $\lim _{t \rightarrow+\infty} V\left(x\left(x_{0}, \phi_{0}, t\right)-\Psi\left(\phi_{0}, t\right), \phi^{x}\left(x_{0}, \phi_{0}, t\right)-\phi^{\Psi}\left(\Psi_{0}, \phi_{0}, t\right), t\right)=0$, а тогда из неравенства $(17)$ следует, что $\lim _{t \rightarrow+\infty}\left\|x\left(x_{0}, \phi_{0}, t\right)-\Psi\left(\phi_{0}, t\right)\right\|=0$.

Теперь справедливость теоремы 3 следует из тождества (10) и предела (11).

Теорема 3 доказана.

В некоторых случаях удобнее пользоваться теоремой 4 , в которой производная (18) допускает бесконечно малый высший предел по совокупности переменных $x, \phi$.

Теорема 4. Если при $\varepsilon=\varepsilon_{0}$ существует $x$-знакоопределеннал функиия $V(x, \phi, t)$, для которой функиия (18) знакоопределеннал, знака, противоположного $\kappa$ знаку $V(x, \phi, t)$, а сама функиия $V(x, \phi, t)$ допускает бесконечно малый высший предел, то при $\nu_{0}=\xi\left(\varepsilon_{0}\right)$ интегральное многообразие $\Psi\left(\phi_{0}, t\right)$ системъ (1) устойчиво асимптотически.

Доказательство теоремы 4 практически не отличается от доказательства теоремы 3.

Определение 4. Функцию $y=\Gamma(\varepsilon, x, \phi, t)$, удовлетворяющую соотношениям (10) и (11), будем называть равномерно возрастающей по $x$, если для любого достаточно малого $\epsilon_{1}>0$ найдется такое $\epsilon_{2}>0$, что из выполнения неравенства $\|x\|>\epsilon_{2}$ следует справедливость $\|\Gamma(\varepsilon, x, \phi, t)\|>\epsilon_{1}$ сразу для всех $\varepsilon, \phi, t$ в области $\mathbb{R}^{m+1} \times \mathrm{X}_{n} \times \mathrm{E}$, где $\epsilon_{2}<\delta_{1}$.

Теорема 5. Если функиия $y=\Gamma(\varepsilon, x, \phi, t)$ равномерно возрастает по $x$ и при $\varepsilon=\varepsilon_{0}$ существует функиия $V(x, \phi, t)$, допускающая бесконечно малый высший предел по переменной $x$, для которой производная (18) $x$-знакоопределенная, а сама функиия $V(x, \phi, t)$ в области $\mathrm{X}_{n} \times \mathbb{R}^{m+1}$ при сколь угодно малой $\delta_{1}$ ограничена и может принимать значения того же знака, что и производная (18), то при $\nu_{0}=\xi\left(\varepsilon_{0}\right)$ интегральное многообразие $\Psi\left(\phi_{0}, t\right)$ системы (1) неустойчиво.

Доказательство. Пусть $\epsilon_{1}>0$-произвольное сколь угодно малое число, $\varepsilon=\varepsilon_{0}$ и $d V / d t-$ положительная. Значит, можно выбрать постоянную $\epsilon_{2}>0$ и начальные данные $x_{0}$ настолько близкими к $\Psi_{0}$, чтобы одновременно выполнялось

$$
\begin{gathered}
\left\|\Gamma\left(\varepsilon_{0}, x_{0}, \phi_{0}, 0\right)-\Gamma\left(\varepsilon_{0}, \Psi_{0}, \phi_{0}, 0\right)\right\| \leqslant \epsilon_{1}, \\
\|\Gamma(\varepsilon, x, \phi, t)\|>\epsilon_{1} \text { при }\|x\|>\epsilon_{2}, V\left(x_{0}-\Psi_{0}, 0,0\right)>0 . \text { Поэтому } \\
\left\|x_{0}-\Psi_{0}\right\| \leqslant \epsilon_{2} .
\end{gathered}
$$

Предположим, что с течением времени неравенство (26) не нарушится. Тогда, так как $d V / d t$ положительная, то

$$
V\left(x\left(x_{0}, \phi_{0}, t\right)-\Psi\left(\phi_{0}, t\right), \phi^{x}\left(x_{0}, \phi_{0}, t\right)-\phi^{\Psi}\left(\Psi_{0}, \phi_{0}, t\right), t\right)>V\left(x_{0}-\Psi_{0}, 0,0\right)
$$

при $t \in(0 ;+\infty)$ и, следовательно,

$$
\left\|x\left(x_{0}, \phi_{0}, t\right)-\Psi\left(\phi_{0}, t\right)\right\| \geqslant \epsilon_{3}>0
$$


Но тогда $d V / d t \geqslant \epsilon_{4}>0$ и, значит,

$$
V\left(x\left(x_{0}, \phi_{0}, t\right)-\Psi\left(\phi_{0}, t\right), \phi^{x}\left(x_{0}, \phi_{0}, t\right)-\phi^{\Psi}\left(\Psi_{0}, \phi_{0}, t\right), t\right) \geqslant V\left(x_{0}-\Psi_{0}, 0,0\right)+\epsilon_{4} t,
$$

что противоречит ограниченности функции $V(x, t)$ в области $(26)$.

Полученное противоречие доказывает, что с течением времени неравенство (26) нарушается и вместе с ним нарушается неравенство $(25)$. Таким образом, интегральное многообразие $\Psi\left(\phi_{0}, t\right)$ системы (1) неустойчиво.

Теорема 5 доказана.

В качестве иллюстрации доказанных выше теорем рассмотрим одну систему вида (1).

Пример. Пусть

$$
\begin{gathered}
\alpha_{1}=\nu_{1}-\nu_{2}+\nu_{3}+\sqrt{y_{1}^{2}+y_{3}^{2}}, \quad \alpha_{2}=2 y_{1}^{2}+3 y_{3}^{2}, \quad \alpha_{3}=\nu_{1}+\nu_{2}-\nu_{3}, \\
\alpha_{4}=\left(\alpha_{2}++\alpha_{3}\right) \alpha_{1} \sqrt{y_{1}^{2}+y_{3}^{2}} y_{2}, \quad \alpha_{5}=\left(\alpha_{3}-\alpha_{2}\right) \alpha_{1} \sqrt{y_{1}^{2}+y_{3}^{2}} y_{2}
\end{gathered}
$$

и рассмотрим систему трех уравнений

содержащую только скалярные величины.

$$
\left\{\begin{array}{l}
\dot{y}_{1}=-\alpha_{4} y_{1} y_{2}-\alpha_{1} y_{3}, \\
\dot{y}_{2}=\alpha_{5} \alpha_{1} \sqrt{y_{1}^{2}+y_{3}^{2}}, \\
\dot{y}_{3}=-\alpha_{4} y_{3} y_{2}+\alpha_{1} y_{1},
\end{array}\right.
$$

Замена $y_{1}=x_{1} \cos \phi, y_{2}=x_{2}, y_{3}=x_{1} \sin \phi, \varepsilon_{i}=\nu_{i}$ приводит уравнения (27) к виду (3)

$$
\left\{\begin{array}{l}
\dot{x}_{1}=-\beta_{1}\left(\varepsilon_{1}, \varepsilon_{2}, \varepsilon_{3}, x_{1}, x_{2}, \phi\right) x_{1}, \\
\dot{x}_{2}=\beta_{2}\left(\varepsilon_{1}, \varepsilon_{2}, \varepsilon_{3}, x_{1}, x_{2}, \phi\right) x_{2}, \\
\dot{\phi}=\varepsilon_{1}-\varepsilon_{2}+\varepsilon_{3}+x_{1},
\end{array}\right.
$$

где

$$
\begin{aligned}
& \beta_{1}=\left(\varepsilon_{1}+\varepsilon_{2}-\varepsilon_{3}+x_{1}^{2}\left(2+\sin ^{2} \phi\right)\right) x_{1} x_{2}^{2}\left(\varepsilon_{1}-\varepsilon_{2}+\varepsilon_{3}+x_{1}\right), \\
& \beta_{2}=\left(\varepsilon_{1}+\varepsilon_{2}-\varepsilon_{3}-x_{1}^{2}\left(2+\sin ^{2} \phi\right)\right) x_{1}^{2}\left(\varepsilon_{1}-\varepsilon_{2}+\varepsilon_{3}+x_{1}\right)^{2} .
\end{aligned}
$$

Тогда уравнения (7) и (8) соответственно запишутся следующим образом:

$$
\begin{gathered}
\dot{\phi}=\varepsilon_{1}\left(\phi_{0}\right)-\varepsilon_{2}\left(\phi_{0}\right)+F_{1}\left(\phi_{0}, t\right), \\
\left\{\begin{array}{l}
\dot{x}_{1}=-\beta_{1}\left(\varepsilon_{1}\left(\phi_{0}\right), \varepsilon_{2}\left(\phi_{0}\right), 0, F_{1}\left(\phi_{0}, t\right), F_{2}\left(\phi_{0}, t\right), \phi_{t}^{F}\right) x_{1}, \\
\dot{x}_{2}=\beta_{2}\left(\varepsilon_{1}\left(\phi_{0}\right), \varepsilon_{2}\left(\phi_{0}\right), 0, F_{1}\left(\phi_{0}, t\right), F_{2}\left(\phi_{0}, t\right), \phi_{t}^{F}\right) x_{2},
\end{array}\right.
\end{gathered}
$$

а матрицант уравнения (8)

$$
\begin{aligned}
Y_{\varepsilon}^{F}\left(\phi_{0}, t\right)=\operatorname{diag}\left[\exp \left(-\int_{0}^{t} \beta_{1}\left(\varepsilon_{1}\left(\phi_{0}\right), \varepsilon_{2}\left(\phi_{0}\right), 0, F_{1}\left(\phi_{0}, \tau\right), F_{2}\left(\phi_{0}, \tau\right), \phi_{\tau}^{F}\right) d \tau\right),\right. \\
\left.\exp \left(\int_{0}^{t} \beta_{2}\left(\varepsilon_{1}\left(\phi_{0}\right), \varepsilon_{2}\left(\phi_{0}\right), 0, F_{1}\left(\phi_{0}, \tau\right), F_{2}\left(\phi_{0}, \tau\right), \phi_{\tau}^{F}\right) d \tau\right)\right] .
\end{aligned}
$$

Выбирая теперь преобразующую матрицу $Q_{\varepsilon}^{F}\left(\phi_{0}\right) \equiv I_{2}$ и $l=1$, приходим к уравнению (13):

$$
\left\{\begin{array}{l}
\varepsilon_{1}\left(\phi_{0}\right)+\varepsilon_{2}\left(\phi_{0}\right)+\frac{1}{2 \pi} \int_{0}^{2 \pi} F_{1}^{2}\left(\phi_{0}, t\right)\left(2+\sin ^{2} \phi_{0}\right) d t=0, \\
\left.\varepsilon_{1}\left(\phi_{0}\right)-\varepsilon_{2}\left(\phi_{0}\right)+\frac{1}{2 \pi} \int_{0}^{2 \pi} F_{1}\left(\phi_{0}, t\right)\right) d t=0 .
\end{array}\right.
$$


Итак,

$$
\begin{aligned}
& \varepsilon_{1}^{F}\left(\phi_{0}\right)=-\frac{1}{4 \pi} \int_{0}^{2 \pi}\left(F_{1}\left(\phi_{0}, t\right)+F_{1}^{2}\left(\phi_{0}, t\right)\left(2+\sin ^{2} \phi_{t}\right)\right) d t, \\
& \varepsilon_{2}^{F}\left(\phi_{0}\right)=\frac{1}{4 \pi} \int_{0}^{2 \pi}\left(F_{1}\left(\phi_{0}, t\right)-F_{1}^{2}\left(\phi_{0}, t\right)\left(2+\sin ^{2} \phi_{t}\right)\right) d t
\end{aligned}
$$

являются единственным решением уравнения (13) для каждой функции $F\left(\phi_{0}, t\right)$. Тогда по теореме 1 у системы (27) существует интегральное многообразие $\Psi\left(\phi_{0}, t\right)$, удовлетворяющее $(16)$. Значит, в данном случае выполняются равенства

$$
\Psi_{1}\left(\phi_{0}, t\right) \equiv c, \quad \Psi_{2}\left(\phi_{0}, t\right) \equiv 0, \quad \phi_{t}^{\Psi} \equiv \phi_{0},
$$

то есть

$$
\varepsilon_{1}^{\Psi}\left(\phi_{0}\right)+\varepsilon_{2}^{\Psi}\left(\phi_{0}\right)=-c^{2}\left(2+\sin ^{2} \phi_{0}\right), \quad \varepsilon_{1}^{\Psi}\left(\phi_{0}\right)-\varepsilon_{2}^{\Psi}\left(\phi_{0}\right)=-c .
$$

Поэтому в системе (28) получим

$$
\begin{gathered}
\beta_{1}=\left(-c^{2}\left(2+\sin ^{2} \phi_{0}\right)+x_{1}^{2}\left(2+\sin ^{2} \phi\right)\right) x_{1} x_{2}^{2}\left(x_{1}-c\right), \\
\beta_{2}=\left(-c^{2}\left(2+\sin ^{2} \phi_{0}\right)-x_{1}^{2}\left(2+\sin ^{2} \phi\right)\right) x_{1}^{2}\left(x_{1}-c\right)^{2} .
\end{gathered}
$$

Выберем функцию $V(x, \phi, t) \equiv x_{1}^{2}+x_{2}^{2}$. Тогда

$$
\frac{d V}{d t}=-2\left(x_{1}-c\right)^{2} x_{1}^{4} x_{2}^{2}\left(2+\sin ^{2} \phi\right) \leqslant 0
$$

(см. (18)). Таким образом, из теоремы 2 следует, что решение $\Psi\left(\phi_{0}, t\right)$ устойчивое. Заметим, что теоремы из [19] здесь применить невозможно, поскольку $d V / d t$ зависит от $\phi$.

Таким образом, нам удалось получить новые достаточные условия существования устойчивых и неустойчивых интегральных многообразий системы (1). При этом задача устойчивости (неустойчивости) по всем переменным $(y)$ решается по свойствам лишь части переменных $(x)$.

\section{СПИСОК ЛИТЕРАТУРЫ}

1. Бибиков Ю. Н. Многочастотные нелинейные колебания и их бифуркации. - Л.: Изд-во ЛГУ, 1991.

2. Боголюбов Н. Н. О некоторых статистических методах в математической физике. - Львов: Изд-во AH УCCP, 1945.

3. Волков Д. Ю. Бифуркация инвариантных торов из состояния равновесия при наличии нулевых характеристических чисел// Вестн. Ленингр. ун-та. - 1988. - 1, № 2. - С. 102-103.

4. Гребенников Е. А., Рябов Ю. А. Конструктивные методы анализа нелинейных систем. - М.: Наука, 1979.

5. Куликов Д. А., Секацкая А. В. О влиянии геометрических характеристик области на структуру нанорельефа// Вестн. Удмурт. ун-та. Мат. Мех. Комп. науки. - 2018. - 28, № 3. - С. 293-304.

6. Купцов М. И. Локальное интегральное многообразие систем дифференциальных уравнений, зависящих от параметра// Диффер. уравн. - 1999. - 35, № 11. - С. 1579-1580.

7. Купцов М. И. Существование интегрального многообразия системы дифференциальных уравнений// Диффер. уравн. - 1998. - 34, № 6. - С. 855.

8. Купцов М. И. Существование интегральных многообразий и периодических решений системы обыкновенных дифференциальных уравнений/ Дисс. на соиск. уч. степ. канд. физ.-мат. наук - Ижевск: Удмурт. гос. ун-т, 1997.

9. Купцов М. И., Минаев В. А., Фаддеев А. О., Яблочников С. Л. К вопросу об устойчивости интегрального многообразия системы дифференциальных уравнений в критическом случае// Тез. докл. Междунар. конф. «Геометрические методы в теории управления и математической физике» (25-28 сентября 2018 г., Рязань). - Рязань: РГУ, 2018. - С. 16-17. 
10. Купцов М. И., Терехин М. Т., Теняев В. В. К проблеме существования интегральных многообразий системы дифференциальных уравнений, не разрешенных относительно производных// Ж. Средневолж. мат. о-ва. - 2017. - 19, № 2. - С. 76-84.

11. Курбаншоев C. З., Нусайриев M. А. Построение оптимальных интегральных многообразий для нелинейных дифференциальных уравнений// Докл. АН Респ. Таджикистан. - 2014. - 57, № 11-12. C. 807-812.

12. Митропольский Ю. А., Гребенников Е. А. Метод усреднения в исследованиях резонансных систем. - М.: Наука, 1992.

13. Митропольский Ю. А., Лъкова О. Б. Интегральные многообразия в нелинейной механике. - М.: Наука, 1973.

14. Неймарк Ю. И. Метод точечных отображений в теории нелинейных колебаний. - М.: Либроком, 2010.

15. Румянцев B. В., Озиранер А. С. Устойчивость и стабилизация движения по отношению к части переменных. - М.: Наука, 1987.

16. Самойленко А. М. Элементы математической теории многочастотных колебаний. Инвариантные торы. - М.: Наука, 1987.

17. Щетинина E. B. Интегральные многообразия и затягивание потери устойчивости/ Дисс. на соиск. уч. степ. канд. физ.-мат. наук - Воронеж: Воронеж. гос. ун-т, 2005.

18. Kuptsov M. I. Local integral manifold of a system of differential equations// Differ. Equ. — 1998. - 34, № 7. - P. 1005-1007.

19. Kuptsov M. I., Minaev V. A. Stability integral manifold of the differential equations system in critical case// J. Phys. Conf. Ser. — 2018. - 973. - 012055.

20. Sobolev $V$. Slow integral manifolds and control problems in critical and twice critical cases// J. Phys. Conf. Ser. - 2016. - 727. — 012017.

21. Vasilina G. K., Tleubergenov M. I. On the optimal stabilization of an integral manifold// J. Math. Sci. 2018. - 229, № 4. - P. 390-402.

22. Yablochnikov S. L., Yablochnikova I. O., Vidov S. V., Kuptsov M. I., Olisaeva A. V. The aspects of modeling information processes realized in complex telecommunication systems// Proc. Conf. "Wave Electronics and Its Application in Information and Telecommunication Systems" (November 26-30, 2018, Saint Petersburg). — IEEE, 2018. - P. 1-5.

Купцов Михаил Иванович

Рязанский государственный радиотехнический университет

E-mail: kuptsov_michail@mail.ru

Минаев Владимир Александрович

Московский государственный технический университет им. Н. Э. Баумана

(национальный исследовательский университет)

E-mail: m1va@yandex.ru

Маскина Мария Сергеевна

Академия права и управления Федеральной службы исполнения наказаний России, Рязань

E-mail: mariya_maskina@mail.ru 